\title{
LA ATENUACIÓN COMO ESTRATEGIA PRAGMÁTICA EN EL MONÓLOGO HUMORÍSTICO SUBVERSIVO'
}

\author{
ATTENUATION AS A PRAGMATIC STRATEGY IN SUBVERSIVE STAND-UP COMEDY
}

\section{Esther Linares Bernabéu}

Universitat d'Alacant

Resumen

La presente investigación analiza la función de la atenuación en el monólogo humorístico representado por las cómicas españolas de la actualidad. Partimos de la hipótesis de que la atenuación es una de las estrategias discursivas empleadas por las cómicas que emplean un humor subversivo, ya que el uso de la atenuación en un contexto humorístico permite que el hablante pueda tratar, con mayor facilidad, temas o asuntos que en principio podrían resultar controvertidos o problemáticos. Con el objetivo de verificar tal conjetura, este estudio recoge un corpus de 15 monólogos humorísticos representados en salas y teatros españoles durante los años 2017 y 2018. La transcripción y segmentación de dichas muestras nos ha permitido extraer la frecuencia de uso de las distintas tácticas y procedimientos lingüísticos de atenuación empleados en el discurso humorístico subversivo, así como examinar las funciones que cumple la atenuación como estrategia pragmática. Los resultados obtenidos certifican que las humoristas españolas analizadas recurren a la atenuación y, por tanto, se distancian lingüísticamente del mensaje, para poder acercarse -o no alejarse demasiado- de su público.

\begin{tabular}{|c|c|c|}
\hline $\begin{array}{l}\text { PALABRAS } \\
\text { humorístico, } \\
\text { discursiva }\end{array}$ & $\begin{array}{l}\text { CLAVE: } \\
\text { humor }\end{array}$ & $\begin{array}{l}\text { atenuación, } \\
\text { subversivo, }\end{array}$ \\
\hline
\end{tabular}

Abstract

This research examines the function of attenuation in the humorous monologue performed by current Spanish female comedians. Our first hypothesis is that attenuation is one of the discursive strategies used by the comedians who represent a subversive type of humor, since the use of attenuation in a humorous context allows the speaker to easier deal with topics or issues that could be controversial or troublesome in the beginning. With the aim of verifying this assumption, this study reflects a corpus of 15 humorous monologues performed in different Spanish auditoriums and theatres during the years 2017 and 2018. The transcription and segmentation of these samples has allowed us to draw the frequency of use regarding the different tactics and linguistic procedures for attenuating the subversive humorous discourse, as well as to examine the functions attenuation fulfils as a pragmatic strategy. The obtained results certify that the Spanish female comedians under review use attenuation and thereby get off the subject linguistically, in order to get closer to the public-or not too much distant from it-.

KEY WORDS: attenuation, stand-up comedy, subversive humor, discourse strategy

\footnotetext{
1 La investigación recogida en el presente artículo ha sido posible gracias al contrato predoctoral financiado por la Conselleria d'Educació, Investigació, Cultura i Esport de la Generalitat Valenciana a través del Proyecto de investigación "Humor de género: Observatorio de la identidad de mujeres y hombres a través del humor" (PROMETEO/2016/052. IP: Leonor Ruiz Gurillo).
} 


\section{INTRODUCCIÓN}

A menudo el humor se asocia como un fenómeno comunicativo propio de la cortesía positiva. De hecho, Brown y Levinson (1987) defienden que el humor está servicio de la cortesía en tanto que contribuye a mantener la solidaridad entre los hablantes y el sentimiento de pertenencia al grupo.

No obstante, existen diversos tipos de humor y no todos tienen como fin principal mantener la imagen positiva ${ }^{2}$ de todos los participantes de la comunicación, entendida esta como la voluntad del interlocutor de ser aceptado por parte del resto de participantes (Brown y Levinson, 1987: 62). De hecho, a través del humor se puede atacar la imagen pública de un determinado colectivo o individuo y, por tanto, dar lugar a situaciones descorteses (Alvarado, 2016: 250). Este sería el caso, por ejemplo, del humor subversivo. De acuerdo con Holmes y Marra (2002), este tipo de humor desafía las relaciones de poder existentes, ya sea de forma explícita o implícita. Así pues, para subvertir el statu quo, los hablantes emplean una serie de estrategias discursivas para atacar y distanciarse socialmente del objeto de la crítica, sin alejarse demasiado de los oyentes e, incluso, creando lazos con determinados sectores.

En este sentido, el objetivo de este trabajo es analizar la función de la atenuación en el monólogo humorístico representado por diversas cómicas españolas. Partimos de la hipótesis inicial de que la atenuación es una de las estrategias discursivas empleadas por las humoristas que representan un humor subversivo con el objetivo de desafiar el heteropatriarcado y romper con determinados estereotipos. De hecho, siguiendo las ideas de Douglas (1968) y Kuipers (2008), consideramos que el uso de la atenuación en un contexto humorístico permite que el hablante pueda tratar, con mayor facilidad, temas o asuntos que en principio podrían resultar controvertidos o problemáticos. Asimismo, como segunda hipótesis general, creemos que los principales mecanismos lingüísticos de atenuación, presentados por Albelda y Cestero, (2011) y Briz y Albelda (2013), están también al servicio del humor y, por tanto, forman parte de los elementos lingüísticos y extralingüísticos propios del discurso humorístico.

Por consiguiente, en las páginas que siguen, estableceremos el marco teórico sobre el que se asentará la investigación acerca de la relación existente entre los fenómenos de la atenuación, la cortesía y el humor. (\$2). Seguidamente, expondremos los instrumentos metodológicos que hemos empleado para la recogida del corpus y la extracción de los datos (§3). El análisis cuantitativo y cualitativo, junto con la discusión de los resultados obtenidos ocuparán la parte central del trabajo (§4). Finalmente, una reflexión sobre el estado actual del tema y las futuras líneas de investigación cerrará este estudio (§5).

\section{MARCO TEÓRICO}

\subsection{La atenuación en el discurso humorístico planificado}

Durante el último medio siglo, diferentes lingüistas se han preocupado por la labor de definir, delimitar y estudiar en distintos géneros la atenuación como categoría pragmática (Zadeh, 1965; Lakoff, 1973; Fraser, 1989; Holmes, 1984; Bravo, 1993; Briz, 1995, 2007 y Albelda, 2008, 2010; Albelda y Cestero, 2011). Para Briz (2007), la atenuación es un

\footnotetext{
${ }^{2}$ Seguimos en este trabajo la concepción de face desarrollada por el sociólogo canadiense Erving Goffman en 1967.
} 
mecanismo lingüístico que tiene que ver siempre con la eficacia, en tanto que contribuye a que el interlocutor acepte los argumentos del hablante, pero no siempre con la cortesía ${ }^{3}$. De hecho, cuando atenuamos para salvaguardar nuestra imagen, no estamos siendo corteses. En este sentido, la atenuación se define como una actividad argumentativa estratégica de debilitación de la fuerza ilocutiva y del papel de los participantes en la enunciación para lograr con éxito la meta prevista (Briz y Albelda, 2013). Es decir, el hablante debilita la fuerza ilocutiva, de tal manera que logra así distanciarse de su mensaje para acercase -social y afectivamente- o no alejarse demasiado del otro o de terceros.

Esta definición enlazaría con la idea de Greenbaum (1999:33) de que el cómico emplea diferentes estrategias narrativas, propias del discurso humorístico, para persuadir y controlar su discurso argumentativo. La autora identifica al monólogo como un discurso propiamente retórico-argumentativo, puesto que, para divertir al público, las cómicas deben buscar el acuerdo con la audiencia. Una de dichas estrategias con las que buscar la aceptación y risa del público sería la atenuación.

\subsection{Factores que fomentan el uso de la atenuación en el monólogo humorístico}

Como veremos a través de los ejemplos de nuestro corpus, el monólogo humorístico no televisado, es decir, aquel que se representa en teatros o bares, representa una especie de diálogo con el público, lo cual fomenta la presencia de rasgos conversacionales como la inmediatez, el cara a cara, la retroalimentación o el dinamismo con la audiencia. Así pues, partiendo de los rasgos situacionales que caracterizan a la conversación coloquial, tanto los primarios como los coloquializadores, propuesto por el grupo Val.Es.Co., veremos cómo la no presencia de cualquiera de ellos promueve el uso de la atenuación.

Tabla 1: Rasgos situacionales

\begin{tabular}{c|c|}
\hline RASGOS PRIMARIOS & RASGOS COLOQUIALIZADORES \\
$\begin{array}{c}\text { 1.1. Conversacionales: } \\
+ \text { Toma de turno no predeterminada } \\
\pm \text { Dinamismo conversacional } \\
\pm \text { Retroalimentación } \\
\quad+\text { Inmediatez }\end{array}$ & \pm Relación de igualdad \\
\pm Relación vivencial \\
1.2. Coloquiales propiamente dichos: \\
\pm Planificación \\
+ Fin interpersonal \\
1.3. Tipológicos \\
+ Cara a cara \\
+ Tono informal
\end{tabular}

Debido a su carácter oral, el monólogo humorístico se configura como un discurso de inmediatez comunicativa (Koch y Oesterreicher, 2007), puesto que se desarrolla en el aquí y el ahora. Asimismo, en este tipo de discurso, no existe una toma de turno predeterminada, ya que el público puede interactuar o responder a lo que dice la cómica en cualquier momento. De hecho, las cómicas le adjudican el papel de hablante al público cuando este responde a sus intervenciones, lo que, a su vez, supone que la monologuista

\footnotetext{
${ }^{3}$ Pese a que la atenuación es uno de los recursos propios de la cortesía en la interacción, no emplear atenuación en géneros discursivos como la conversación coloquial no implica ser menos cortés o descortés. 
vaya adaptando su discurso en relación a dichas respuestas (Rutter, 2001: 308; Ruiz Gurillo, 2013: 202).

Ahora bien, en aras de conseguir con éxito el divertimento del público -principal fin interpersonal de este tipo de discurso-, el monólogo humorístico tiene una alta carga de planificación previa a la dramatización (Ruiz Gurillo, 2013). Es decir, la cómica o el cómico, de forma consciente, organiza y estructura su discurso teniendo en cuenta su posible público y el efecto que sus palabras pueden causar (Rutter, 2001). Asimismo, este tipo de discurso humorístico planificado presenta diferencias con relación a los rasgos coloquializadores típicos de la conversación cotidiana. De hecho, el monologo no se desarrolla en un marco interactivo cotidiano, sino que existe un escenario con un pie de micro, un taburete y unas luces enfocando a la cómica. Así pues, pese a que la monologuista interaccione con el público, e incluso le tutee, no existe una relación de igualdad entre los participantes. Es la humorista quien tiene el control sobre el discurso y maneja los tiempos y temas que tratar, es decir, mantiene una cierta autoridad (Greenbaum, 1999; Yus, 2016). Por otro lado, la aparición de elementos atenuantes viene dada por una relación vivencial parcial, ya que existen saberes compartidos con los que se identifica el público femenino, pero que desconoce el sector masculino. Asimismo, de acuerdo con Albelda (2010), los mensajes con temática conflictiva, la expresión de desacuerdo o de disconformidad son un factor que favorece el uso de la atenuación, puesto que "pueden entrañar un potencial de amenaza para alguna de las imágenes de las personas implicadas" (Albelda, 2010: 53). En este sentido, la temática que se suele tratar en el discurso humorístico subversivo, aun siendo cotidiana, también fomenta el uso de elementos mitigadores puesto que muchos de los temas sobre los que gira el monólogo son tabúes en nuestra sociedad o, simplemente, no se suelen tratar en el discurso público.

\subsection{Tácticas, procedimientos y funciones de la atenuación en el discurso humorístico}

Por otro lado, es necesario mencionar cuáles son las tres principales funciones de la atenuación siguiendo la propuesta de Briz y Estellés (2010) y Briz (2011). Por un lado, se encontraría la función de autoprotección o de salvaguardar el yo, se trataría, pues, de una atenuación autocéntrica, que no estaría relacionada con la cortesía y que se emplearía para ganar o no perder la imagen, así como para evitar o minorar responsabilidades. Ahora bien, debemos señalar que la imagen que las cómicas protegen no es la suya propia como individuo, sino también la que ha construido en esa situación escénica en su rol de monologuista (Bravo, 2002; Zimmerman, 2003; Fuentes Rodríguez, 2016). Por otro lado, podríamos identificar la función de prevención, es decir, cuando el hablante recurre a la atenuación para prevenir posibles desacuerdos y, así, salvaguardar la imagen tanto propia como del oyente. Por último, se encontraría la función de reparación, con la que el hablante intenta corregir o enmendar lo dicho y reparar los daños ya causados en la imagen.

Estas funciones se ejecutan a través de dos principales tácticas de atenuación. Por un lado, se encontraría la despersonalización u ocultación del sujeto, la cual se hace a su vez efectiva a través de formas impersonales gramaticales como se, uno, el nosotros de modestia o el tú, por medio de generalizaciones en las que se alude a la sociedad en general, de construcciones que esconden el agente por medio de la voz pasiva o de nominalizaciones, de los evidenciales y del estilo directo, por medio del cual, la monologuista le da voz a otro locutor para eximir su responsabilidad ante lo dicho (Albelda y Cestero, 2011). 
Por otro lado, la segunda táctica principal en la atenuación sería la relativización de lo dicho o minorización de la fuerza ilocutiva. Esta maniobra se efectúa a través de distintos procedimientos lingüísticos y paralingüísticos como la ironía, que permite esconder la verdadera intención del emisor (Briz, 2005: 56), los diminutivos ${ }^{4}$, los eufemismos, las metáforas, las construcciones suspendidas, las reformulaciones e, incluso, las risas como elemento paralingüístico cuando el hablante se ríe mientras habla para así señalar que está en modo humorístico y reducir la fuerza del acto de habla (Bravo, 1993; Cestero, 1996)

En este sentido, siguiendo las propuestas de Albelda y Cestero (2011) y Briz y Albelda (2013), hemos confeccionado la siguiente plantilla con los principales mecanismos lingüísticos al servicio de la atenuación. Así pues, estos son los veinte procedimientos de atenuación que se han tenido en consideración a la hora de analizar el corpus de monólogos humorísticos y de extraer conclusiones acerca del funcionamiento de la atenuación como estrategia pragmática en este tipo de género discursivo.

Tabla 2 Recursos lingüísticos de atenuación

\begin{tabular}{|c|c|}
\hline OCULTACIÓN DEL SUJETO & RELATIVIZACIÓN O \\
(DESPERSONALIZACIÓN) & INDETERMINACIÓN DE LO \\
EXPRESADO & Diminutivos \\
\hline Impersonalización gramatical & Cuantificadores minimizadores, \\
Generalizaciones & aproximativos o difusores \\
Evidenciales & Eufemismos \\
Estilo directo & Ironía \\
& Metáforas \\
Lítotes \\
& Palabras extranjeras \\
Marcadores discursivos \\
Proformas deícticas \\
Construcciones suspendidas \\
Reformulaciones \\
Verbos performativos \\
Estructuras causales \\
Modalizadores de los tiempos verbales \\
Disculpas \\
Entre risas
\end{tabular}

\section{CORPUS Y METODOLOGÍA DE ANÁLISIS}

Siguiendo las bases teóricas y metodológicas para el análisis de la atenuación en corpus discursivos orales propuestos por la bibliografía (Albelda y Cestero, 2011; Briz y Albelda, 2013; Albelda et al., 2014), hemos analizado un corpus que cuenta con un total de 15 monólogos, recogidos durante los años 2017 y 2018 en distintos locales y teatros del territorio español. Estos monólogos han sido representados por 15 mujeres humoristas del panorama de la comedia actual. En concreto, hemos analizado el discurso de las cómicas Nuria Jiménez, Esther Gimeno, Eva Soriano, Silvia Sparks, Pilar de Francisco, Sil de Castro,

\footnotetext{
${ }^{4}$ Ahora bien, no siempre todo sufijo apreciativo atenúa. De hecho, de acuerdo con Briz y Albelda (2013: 300), "el valor atenuante no puede establecerse a partir del acto de habla en que se integra el sufijo apreciativo, sino a partir de un contexto". Normas (ISSN: 2174-7245) | 
Eva Cabezas, Virginia Riezu, Patricia Sornosa, Patricia Espejo, Valeria Ros, Pamela Palenciano, Raquel Sastre, Coria Castillo y Susi Caramelo.

Cada uno de estos monólogos ha sido dividido en secuencias humorísticas, entendiéndose estas como una serie de intervenciones de la monologuista que giran en torno a un tema concreto y que son interrumpidas por las risas, aplausos e, incluso, comentarios del público (Val.Es.Co., 2014: 22-23; Ruiz Gurillo, 2017). En este sentido, hemos transcrito, siguiendo el sistema de transcripción Val.Es.Co. ${ }^{5} 364$ secuencias. Ello supone un total de 660 minutos -11 horas de grabación- y 81.643 palabras.

De estas 364 secuencias, hemos profundizado en aquellas en las que se aprecia un uso de elementos atenuantes, es decir, en 294 secuencias. Como refleja el siguiente gráfico, las secuencias en las que hemos identificado procedimientos atenuantes representan un $80 ` 8 \%$ del total. Ello confirma la notoria presencia de la atenuación como estrategia pragmática y retórica en el discurso humorístico subversivo.

\section{Recuento de ATENUACIÓN}

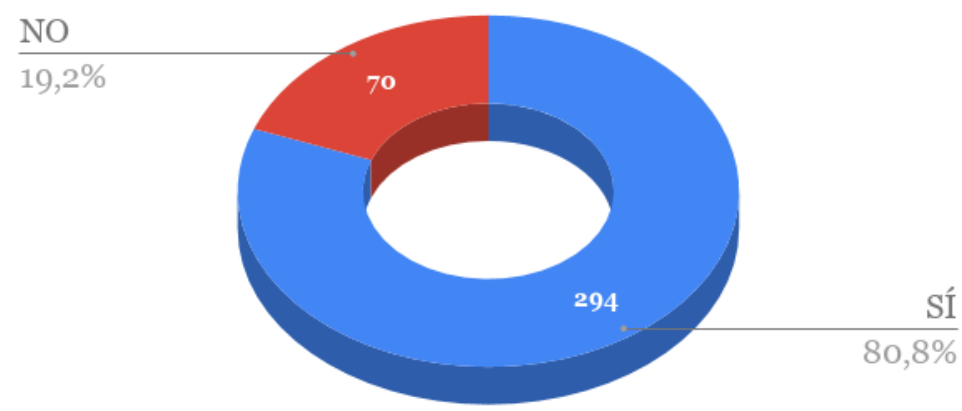

Gráfico 1 Uso de la atenuación en el corpus analizado

\section{ANÁLISIS DE LOS RESULTADOS}

\subsection{Temática que favorece el empleo de la atenuación}

Aunque el elemento de la comunicación que propicia en mayor medida el uso de la atenuación son los interlocutores, en este caso el público ante el que se representa el monólogo, el tema del mensaje también influye en su uso. Como ya comentábamos anteriormente, la temática de este tipo de monólogos propicia la atenuación en aras de no dañar la imagen de los participantes de la situación comunicativa. En el siguiente gráfico hemos analizado el tema principal de cada una de las 294 secuencias en las que se apreciaban rasgos atenuantes:

\footnotetext{
${ }^{5}$ Pueden consultarse las claves del sistema de transcripción Val.Es.Co. en Briz y Grupo Val.Es.Co. (2002: 28-38) o a través del siguiente enlace: https://www.uv.es/valesco/sistema.pdf 


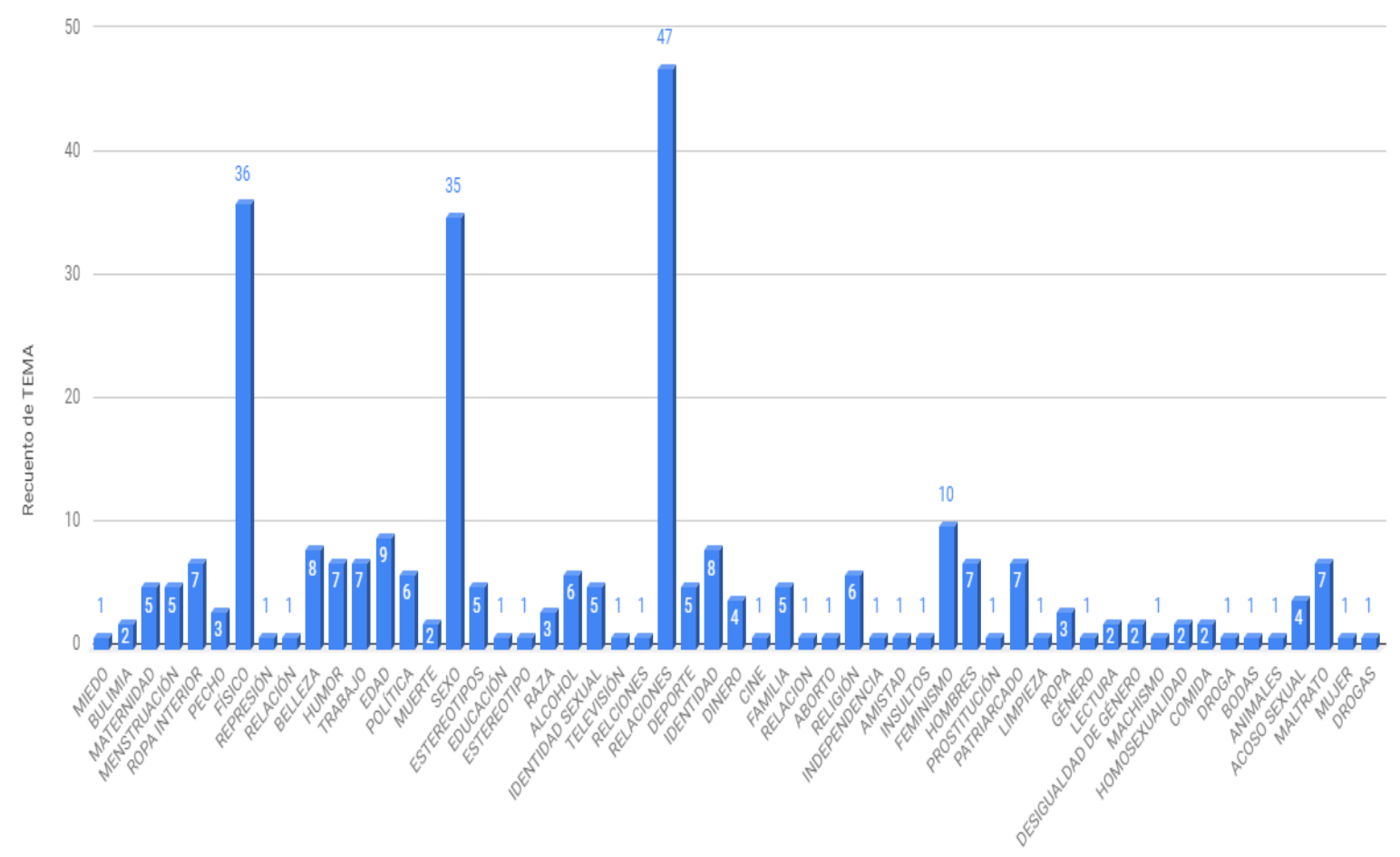

Gráfico 2 Temas de las secuencias analizadas

Vemos, claramente, cómo hay tres temas claves sobre los que giran la mayoría de secuencias humorísticas, estos son: las relaciones de pareja -tanto de amor como de desamor-, el sexo y el aspecto físico. Asimismo, otros temas frecuentes serían el feminismo, el patriarcado, la identidad, la edad y la belleza. Estos resultados refuerzan nuestra hipótesis de partida de que el humor fomenta que el hablante pueda tratar asuntos conflictivos sin parecer ofensivo. Además, otra razón que influye en que se puedan abordar estas temáticas es el hecho de que son monólogos no televisados, y por tanto se representan ante un público, que por lo general está predispuesto a escuchar y reírse sobre esos asuntos (Ruiz Gurillo y Linares Bernabéu, 2018).

\subsection{Tácticas y recursos de atenuación}

Siguiendo la propuesta de Briz y Albelda (2013), los recursos lingüísticos al servicio de la atenuación están, a su vez, relacionados con las dos principales tácticas de atenuación que hemos mencionado previamente en el apartado 2.3, esto es, la ocultación del sujeto y la relativización o indeterminación de lo expresado. 


\section{TÁCTICAS}

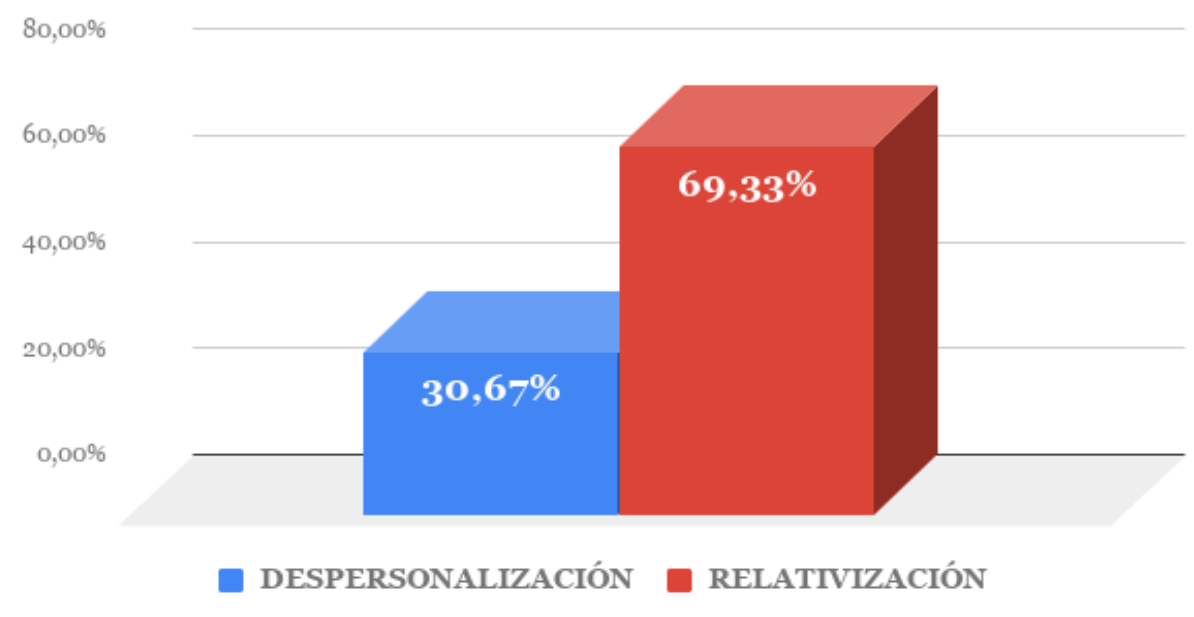

Gráfico 3 Frecuencia de las tácticas de atenuación

Así pues, si analizamos qué táctica de atenuación se lleva a cabo con mayor frecuencia, nos encontramos con que en el 69,33\% de las secuencias analizadas las humoristas tienden a relativizar, mientras que solo en el 30,67\% de los casos emplean tácticas de despersonalización. Ahora bien, cabe decir que estos resultados no nos extrañan demasiado, puesto que existen más recursos lingüísticos relacionados con técnicas de relativización (véase tabla 2).

Por otro lado, atendiendo a la taxonomía de procedimientos lingüísticos de atenuación propuesta por Albelda y Cestero (2011), así como la clasificación realizada por Briz y Albelda (2013), hemos examinado con qué frecuencia se emplean los veinte procedimientos de atenuación enumerados previamente. El siguiente gráfico muestra cuáles son los elementos lingüísticos con función atenuadora más recurrentes en nuestro corpus de monólogos humorísticos representados por cómicas españolas.

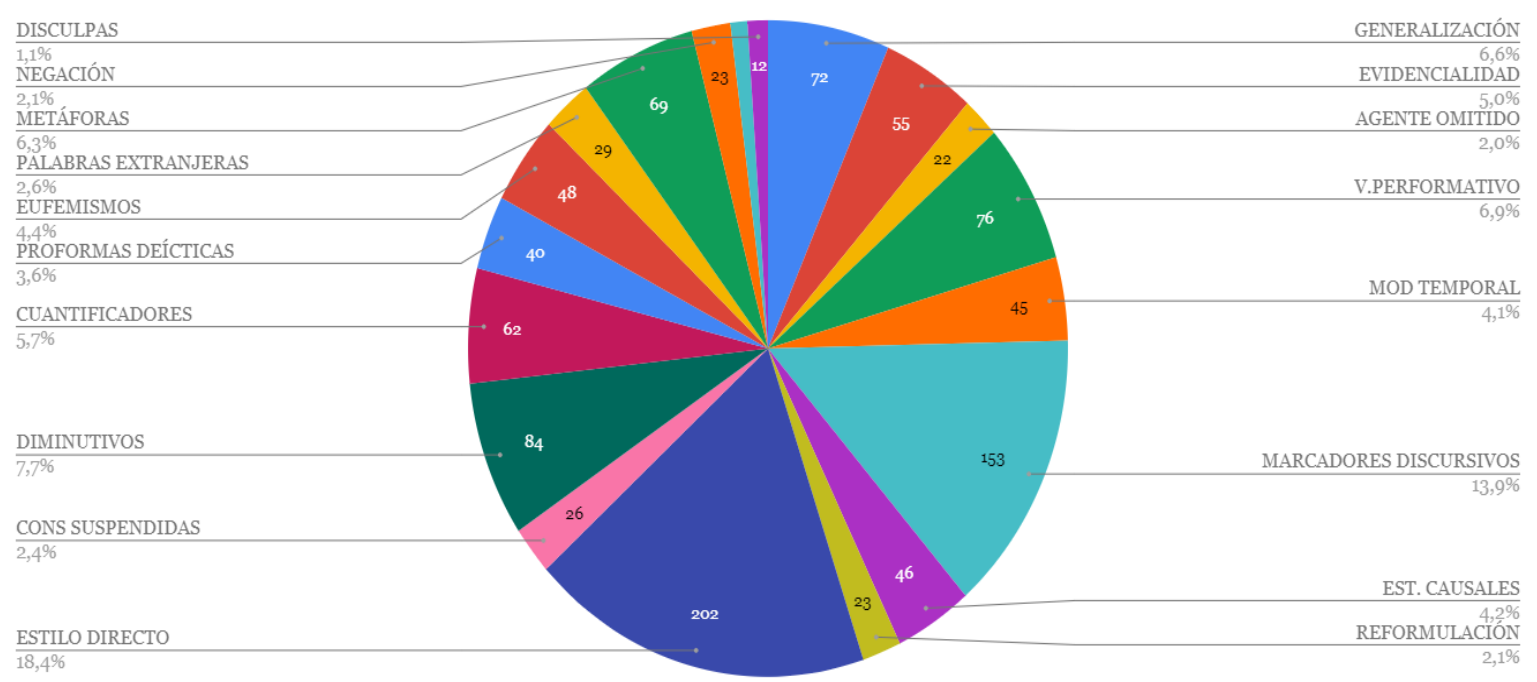

Gráfico 4 Procedimientos lingüísticos de atenuación empleados en el corpus 
Ahora bien, si sacamos la frecuencia de cada uno de los recursos analizados individualmente, el estilo directo es el procedimiento más empleado $(18,4 \%)$, puesto que, como hemos demostrado en investigaciones previas (Linares Bernabéu, en prensa), el discurso directo no solo favorece un distanciamiento lingüístico del mensaje, sino que fomenta el efecto humorístico y la construcción de una identidad de género. Asimismo, observamos cómo los diminutivos (7,7\%), las metáforas (6,3\%) o los cuantificadores $(5,7 \%)$ son también elementos lingüísticos recurrentes en este tipo de discurso y tienen una función dual, ya que sirven tanto como procedimiento de atenuación como indicador lingüístico del humor (Ruiz Gurillo, 2012). Además, resulta también interesante observar el porcentaje de marcadores discursivos (13,9\%), ya que en muchas ocasiones estos se corresponden con marcadores de control de contacto $^{6}$-muy comunes en este tipo de discurso-para buscar la aceptación y el acuerdo con el público. En definitiva, estos resultados vendrían a corroborar nuestra segunda hipótesis en la que defendíamos que los mecanismos lingüísticos de atenuación están también al servicio del humor y, por tanto, forman parte de los elementos lingüísticos y extralingüísticos propios del discurso humorístico.

\subsection{Análisis de las funciones atenuadoras en el monólogo humorístico subversivo}

De acuerdo con las principales investigaciones en atenuación lingüística (Albelda y Cestero, 2011, Briz y Albelda, 2013, Albelda, et al. (2014), para el reconocimiento de la atenuación como categoría pragmática es necesario tener en cuenta el contexto interactivo concreto en el que se produce, esto es, el miembro del discurso causante o desencadenante, la expresión atenuante concreta y el elemento atenuado. De esta forma, el investigador o investigadora podrá decidir si la intención del hablante es minimizar la fuerza ilocutiva del acto de habla o si, por el contrario, se trata de una duda real. Así pues, teniendo esto en consideración, pasaremos a continuación a observar diferentes ejemplos de secuencias humorísticas extraídas del corpus analizado, con el fin de identificar qué función atenuadora realiza el hablante y qué estrategias y procedimientos lingüísticos emplea para ello.

La siguiente secuencia corresponde a un monólogo de la monologuista valenciana Patricia Espejo que fue representado en el local La Cuna en Elche. En concreto, en este fragmento, la cómica le cuenta al público que ha roto con su novio e intenta justificarse de por qué ha decidido dejarlo:

(1)

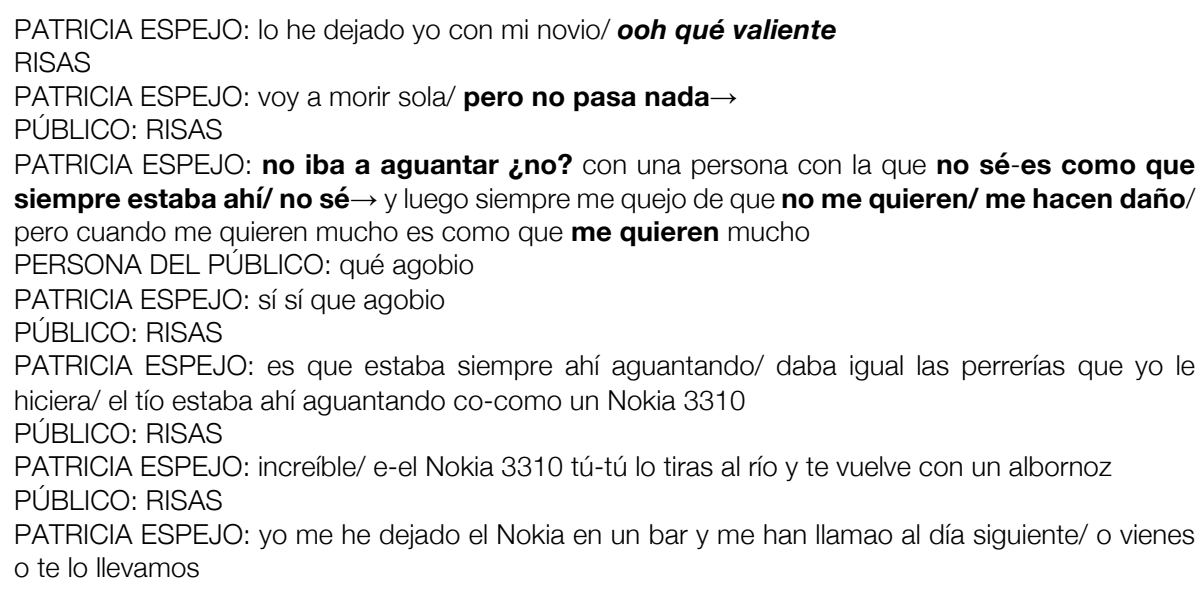

\footnotetext{
${ }^{6}$ Para más información acerca de los marcadores de control de contacto, véase Martín Zorraquino y Portolés (1999). 
PÚBLICO: RISAS

Por un lado, vemos cómo la monologuista reduce la fuerza argumentativa de lo dicho "qué valiente" a través del uso del estilo directo, ya que, al dar voz a otro locutor, se resta responsabilidad ante lo dicho. Asimismo, atenúa la aserción "voy a morir sola" a través del movimiento concesivo "pero no pasa nada", incongruencia que, a su vez, fomenta la risa del público. Por otro lado, atenúa la fuerza ilocutiva de la expresión "no iba a aguantar con una persona que siempre está ahí" por medio de la expresión de duda "no sé" con entonación suspendida, reduciendo así el compromiso epistémico del hablante hacía aquello que sabe con certeza. Además, el uso del imperfecto como modificador del tiempo verbal "iba" y del marcador de control de contacto "¿no?" colaboran también a rebajar el acto de habla. De igual forma, cabe también destacar la táctica de despersonalización que realiza la humorista a través de la omisión del agente y la partícula como cuando dice "y luego siempre me quejo de que no me quieren/ me hacen daño/ pero cuando me quieren mucho es como que me quieren mucho" con el objeto de no hacer referencia a nadie en particular. De igual modo, el efecto humorístico se efectúa con éxito gracias a los distintos indicadores y marcas humorísticas empleadas en la secuencia. Entre ellos, se encontraría la comparación hiperbólica que realiza la cómica entre su novio y el Nokia 3310.

A continuación, el segundo fragmento pertenece a un monólogo de Nuria Jiménez, representado en el local La flauta mágica de Madrid, en el cual la cómica habla de la escasez de variedad en la ropa interior masculina:

(2)

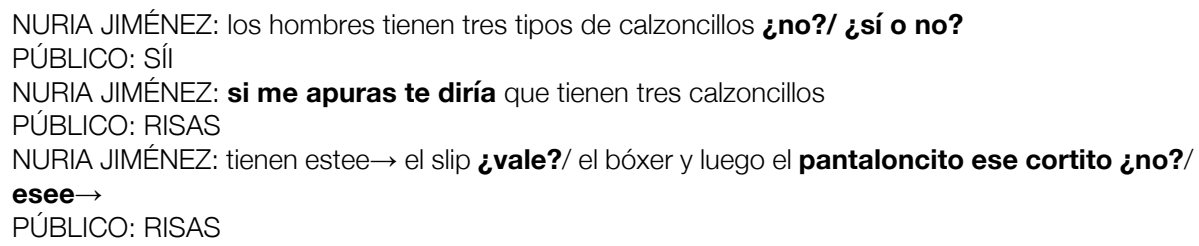

Al situar como objeto de burla al sector masculino, la cómica emplea diferentes recursos de atenuación para hacer su discurso más aceptable. De hecho, vemos cómo emplea los marcadores de control de contacto "¿no?”, "¿sí o no? y “¿vale? para implicar al público en lo dicho y buscar su aprobación. Además, atenúa la aserción "tienen tres calzoncillos", e intenta prevenir posibles daños en la imagen del público masculino, a través de la estructura de restricción "si me apuras" y el uso del verbo en condicional "te diría". Esta afirmación hiperbólica que genera la risa del público se continua con la posterior enumeración de los tipos de calzoncillos, la cual viene también marcada con procedimientos de atenuación lingüística como las proformas deícticas "este" y "ese" con entonación suspendida, así como a través del uso del diminutivo en las palabras "pantaloncito" y "cortito", estando también este último recurso al servicio del efecto humorístico (Ruiz Gurillo, 2014: 150).

De igual modo, nos parece también interesante presentar el siguiente ejemplo, perteneciente a un monólogo de Patricia Sornosa representado en el local El taller tumbao de Alicante. En la secuencia que sigue a continuación, la humorista alude a la sociedad patriarcal en la que vivimos y critica el trato que reciben muchas mujeres:

(3)

PATRICIA SORNOSA: no lo puedo evitar/ miro a mi alrededor y veo que la sociedad trata a las mujeres como si fueran perros $\downarrow$ / LA SOCIEDAD he dicho ¿eh? no los hombres/ podéis relajar el ojete

PÚBLICO: RISAS 


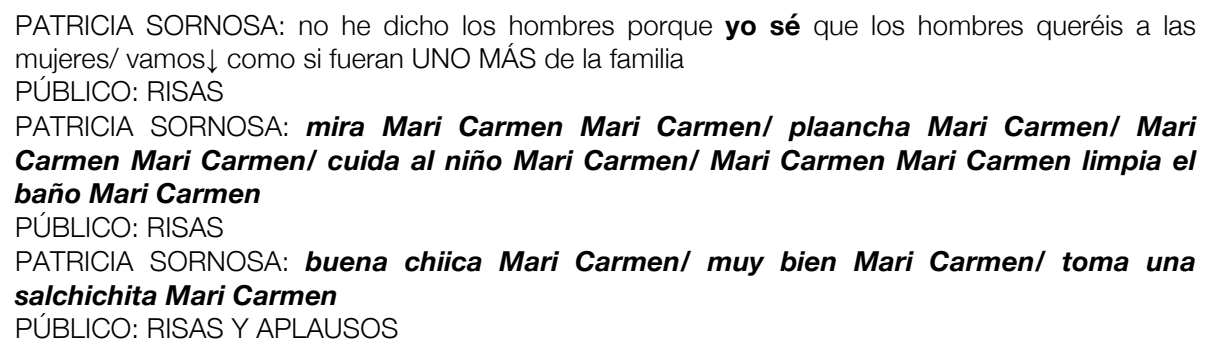

Con la intención de autoproteger su propia imagen y prevenir posibles daños en la imagen de algunos de los allí presentes, la monologuista elude parcialmente su responsabilidad sobre lo que va a decir al afirmar que no lo puede evitar, como si no estuviera en su poder. Además, hace un uso consciente de la expresión generalizadora "la sociedad" para evitar un posible malestar entre los hombres del público y, de hecho, se lo advierte abiertamente a los hombres allí presentes como se observa con el marcador de control de contacto "¿eh?”. Por otro lado, nos parece interesante señalar el acto de habla cortés con fines descorteses (Kaul, 2006) por medio de la expresión irónica "yo sé que los hombres queréis a las mujeres / vamos $\downarrow$ como si fueran UNO MÁS de la familia", de modo que la ironía colabora en la atenuación del mensaje y promueve la risa entre los allí presentes. Así pues, en este caso, la ironía tendría un efecto positivo, ya que sirve para reflejar la opinión contraría del hablante de forma humorística y así, evitar daños en la imagen de algunos miembros del público (Alvarado Ortega, 2009; Pano Alamán, 2018). Por último, el estilo directo, el diminutivo y la metáfora estarían tanto al servicio de la atenuación como del humor. Vemos, por ejemplo, como el estilo directo permite a la cómica desfocalizar los elementos de la enunciación personal y, por otro lado, recurre a la expresión eufemística "salchichita" en aras de causar el divertimiento en el público.

Finalmente, la siguiente secuencia representada por la cómica Eva Cabezas en el teatro Flumen de Valencia aborda uno de los temas más recurrentes del corpus analizado, el aspecto físico y, más concretamente, el peso:

(4)

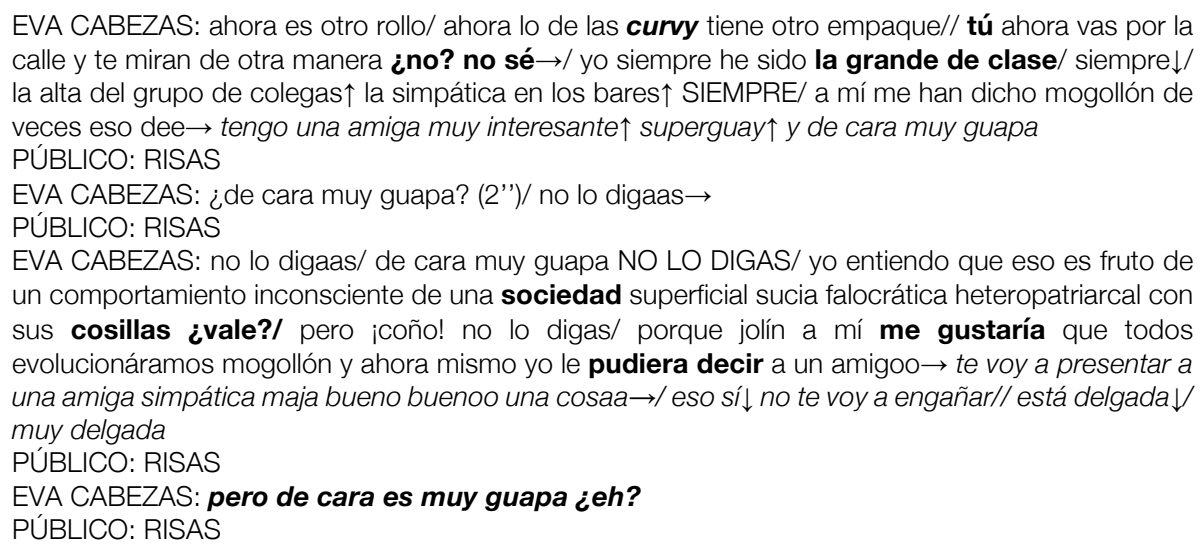

En este caso, la humorista se revela ante la superficialidad de la sociedad actual y critica la importancia que se le da al aspecto físico en nuestra cultura. En concreto, habla de su peso y trata la gordura con diferentes mecanismos de atenuación. Así pues, en este caso, la atenuación tendría principalmente una función autoprotectora. En primer lugar, vemos cómo la cómica emplea el eufemismo "grande" y la palabra extranjera "curvy" para difuminar el contenido semántico de la palabra "gorda", este cambio de código provoca, a su vez, la risa del público. Seguidamente, Eva Cabezas emplea el tú impersonal para ocultar 
su opinión bajo un interlocutor general y, así, atenuar su discurso. Asimismo, el fingimiento de ignorancia, a través la expresión "no sé" con entonación suspendida, colabora a rebajar la fuerza ilocutiva del acto de habla. Otros recursos relativizadores serían los marcadores de control de contacto como "¿no?, ¿vale? o ¿eh?" que contribuyen a relativizar lo dicho previamente, el uso del diminutivo en la expresión "con sus cosillas". Por último, la construcción hipotética que promueve el efecto humorístico emplea el condicional y el subjuntivo como mecanismos de atenuación. Además, la risa del público se genera cuando la monologuista crea conciencia sobre el uso de la estructura concesiva "pero de cara es muy guapa" con valor atenuador, a la hora describir a una chica gruesa, y la subvierte al aplicarla, en una situación hipotética, a las chicas delgadas.

\section{CONCLUSIONES}

Este trabajo ha intentado examinar el funcionamiento de la atenuación en el monólogo humorístico como género discursivo y, más concretamente, en el discurso subversivo que dramatizan las cómicas españolas de la actualidad. Los resultados reflejan que, en este tipo de contexto, predomina la función de autoprotección de la propia imagen y de prevención de daños en la imagen del público. De modo que las humoristas se distancian lingüísticamente del mensaje para poder acercarse -o no alejarse demasiado- de su audiencia.

Por un lado, los datos extraídos reflejan una clara presencia de la atenuación como estrategia pragmática y retórica en el discurso humorístico subversivo, ya que está presente en el $80,8 \%$ de las secuencias del corpus analizado. Por otro lado, hemos comprobado que la atenuación incide en el discurso subversivo reduciendo el papel de los participantes en la enunciación $(30,67 \%)$ y, sobre todo, minimizando la fuerza ilocutiva de los actos de habla (69,33\%). Asimismo, hemos visto cómo los principales recursos lingüísticos atenuantes sirven también, en ocasiones, como elementos humorísticos y coinciden con muchos de los indicadores y marcas del humor propuestos por el grupo GRIALE (Ruiz Gurillo, 2012).

En definitiva, el análisis llevado a cabo en la presente investigación permite corroborar nuestra hipótesis de partida y, así, confirmar que la atenuación es una estrategia retóricopragmática que permite que las monologuistas traten, con mayor facilidad, temas 0 asuntos que en principio podrían resultar controvertidos o problemáticos. Se trataría, pues, de un recurso del que se sirven las cómicas para alcanzar con éxito la meta prevista, que no deja de ser, al fin y al cabo, divertir al público.

\section{REFERENCIAS BIBLIOGRÁFICAS}

Albelda Marco, Marta (2008): «Influence of situational factors in the codification and interpretation of impoliteness», Pragmatics, 18, 4, 751-773.

Albelda MARCo, Marta (2010): «¿Cómo se reconoce la atenuación? Una aproximación metodológica basada en el español peninsular hablado», en Franca Orletti y Laura Mariottini, eds., (Des)cortesía en español. Espacios teóricos y metodológicos para su estudio, Roma, Università degli Studi Roma Tre/Programa EDICE, 47-70.
Albelda Marco, Marta y Ana M. Cestero Mancera (2011): «De nuevo, sobre los procedimientos de atenuación», Español actual, 96, 9-40.

Albelda Marco, Marta, Antonio Briz, Ana Cestero, Dorota KotWICA, y Cristina VILLALBA (2014): «Ficha metodológica para el análisis pragmático de la atenuación en corpus discursivos del español (ES. POR. ATENUACIÓN)», Oralia, 17, 7-62.

Alvarado Ortega, María Belén (2009): «Ironía y cortesía», en Ruiz Gurillo L. y X. Padilla, eds., Dime cómo ironizas y te diré quién eres: una 
aproximación pragmática a la ironía, Frankfurt, Peter Lang, 333-345.

Alvarado Ortega, María Belén (2016): «Descortesía y humor fallido en conversaciones entre hombres y mujeres», Pragmática

Sociocultural/Sociocultural Pragmatics, 4(2), 243-267.

Bravo, Diana (1993): La atenuación de las divergencias mediante la risa en negociaciones españolas y suecas, Estocolmo, Biblioteca de la Universidad de Estocolmo.

BrAvo, Diana. (2002): «Actos asertivos y cortesía: Imagen del rol en el discurso de académicos argentinos», en Plasencia, M.E. y D. Bravo, eds., Actos de habla y cortesía en español, Londres, Lincom, 141-174.

BRIZ GÓmEZ, Antonio (1995): «La atenuación en la conversación coloquial. Una categoría pragmática», en Cortés, L., ed., El español coloquial: actas del I Simposio sobre análisis del discurso oral, Almería, Universidad de Almería, Servicio de Publicaciones, 103-122.

Briz Gómez, Antonio y Grupo VAL.Es.Co. (2002): Corpus de conversaciones coloquiales, Anejo de la revista Oralia, Madrid, Arco-Libros

BRIz Gómez, Antonio (2003): «La estrategia atenuadora atenuadora en la conversación cotidiana española», en Bravo, D., ed., Actas del Primer Coloquio del programa Edice. La perspectiva no etnocentrista de la cortesía: identidad sociocultural de las comunidades hispanohablantes, Estocolmo, Universität Stockholm, 17-46.

BRIZ GÓMEZ, Antonio (2005): «Eficacia, imagen social e imagen de cortesía. Naturaleza de la estrategia atenuadora en la conversación cotidiana española», Estudios de la (des) cortesía en español, 53-91.

BRIz GÓmEZ, Antonio (2007): «Para un análisis semántico, pragmático y sociopragmático de la cortesía atenuadora en España y América», LEA, 19, 1-38.

BrIz GómEZ, Antonio (2010): «Lo coloquial y lo formal, el eje de la variedad lingüística», De moneda nunca usada: Estudios dedicados a José M. ${ }^{a}$ Enguita Utrilla, Zaragoza, Instituto Fernando el Católico/csic, 125-133.

Briz GómEZ, Antonio (2012): «La (no) atenuación y la (des) cortesía, lo lingüístico y lo social: ¿son pareja?», en Escamilla Morales, J. y G. Henry Vega, eds., Miradas multidisciplinares a los fenómenos de cortesía y descortesía en el mundo hispánico, Barranquilla, Universidad del Atlántico/Programa EDICE, 33-75.

Briz Gómez, Antonio, y M. Albelda Marco (2013): «Una propuesta teórica y metodológica para el análisis de la atenuación lingüística en español y portugués: La base de un proyecto en común (ES. POR. ATENUACIÓN)», Onomázein: Revista de lingüística, filología y traducción de la Pontificia Universidad Católica de Chile, 28, 288-319.

BRIz GÓmEZ, Antonio, y María EstelLÉS (2010): «On the relationship between attenuation, discourse particles and position», Studies in Pragmatics, 9, 289-304.

Brown, Penelope y Stephen C. LEVINSON (1987): Politeness: Some universals in language usage (Vol. 4), Cambridge, Cambridge University Press.

Cestero Mancera, Ana María (1996): «Funciones de la risa en la conversación en lengua española», Lingüística Española Actual, 28, 2 , 279-289.

FLORES-TREVIÑo, María Eugenia (2013): «Ironización y atenuación en El Habla de Monterrey PRESEEA» en Martínez, M. L. y J. Castillo, eds., Memorias del VI Coloquio de Humanidades Diálogos sobre Educación, Arte, Cultura y Sociedad, Nuevo León, Fac. de Filosofía y Letras de la UANL.

FRASER, Bruce (1980): «Conversational mitigation», Journal of Pragmatics, 4, 341-350

Fuentes RODRíGueZ, Catalina (2016): «(Des)cortesía, imagen social e identidad como categorías sociopragmáticas en el discurso público», en Domitrescu, Domnita y Diana Bravo, Roles situacionales, interculturalidad y multiculturalidad en encuentros en español, Buenos Aires, EDICE/Dunken,165-192.

GOFFMAN, Erving (1967): Interactional ritual: Essays on face-to-face behavior, New York, Doubleday.

GreENBAUM, Angela (1999): «Stand-up comedy as rhetorical argument. An investigation of comic culture», Humor, 12-1, 33-46.

Grupo VAL.Es.Co.

(2014):

«Las unidades del discurso oral. La propuesta Val.Es.Co. de segmentación de la conversación (coloquial)», Estudios de Lingüística del Español, 35, 13-73.

HOLMES, Janet (1984): «Modifying illocutionary force», Journal of Pragmatics, 8, 345- 365.

HOLMES, Janet (2000): "Politeness, power and provocation: How humour functions in the workplace», Discourse studies, 2(2), 159-185.

HOLMES, Janet., \& Meredith MARRA (2002): «Over the edge? Subversive humor between colleagues and friends", Humor, 15(1), 65-88.

Kaul De Marlangeon, S. (2006): «Tipología del comportamiento verbal descortés en español», en Briz et al., eds., Cortesía y conversación. De lo escrito a lo oral. Actas del III Coloquio Internacional del Programa EDICE, Valencia, Universidad de Valencia, 254-266.

$\mathrm{KoCH}$, Peter y Wulf OesteRREICHER (2007): Lengua hablada en la Romania: español, francés, italiano, Madrid, Gredos

KUIPERS, Giselinde (2008): "The sociology of humor», The primer of humor research, 8, 361398. 
LAKOFF, Robin (1973): «The logic of politeness; or, minding your p's and q's", en Papers from the Regional Meeting, Chicago Linguistic Society 9, Chicago, Chicago Linguistic Society, 292305.

LINARES BERNABÉU, Esther (en prensa): «El estilo de habla en el discurso directo como estrategia para la construcción del género en el monólogo humorístico», Revista Signos.

MARTín ZORRAQUINO, María y José PORTOLÉs LÁZARO (1999): «Los marcadores del discurso» en Bosque, Ignacio y Violeta Demonte, eds., Gramática descriptiva de la lengua española, Madrid, Espasa-Calpe, 4051-4207.

NorRICK, Neal. R., y Alice Spitz (2008): «Humor as a resource for mitigating conflict in interaction", Journal of Pragmatics, 40 (10), 1661-1686.

PANO ALAMÁN, Ana (2018): «Opinión y atenuación en los comentarios de la prensa digital española», Círculo de lingüística aplicada a la comunicación, (73), 103-124.

RuIz GuRILlo, Leonor (2012): La lingüística del humor en español, Madrid, Arco Libros.

RuIz GuRILLO, Leonor (2013): «El monólogo humorístico como tipo de discurso. El dinamismo de los rasgos primarios», Cuadernos Aispi, (2), 195-218.

RuIz GuRILLO, Leonor (2014): «Infiriendo el humor. Un modelo de análisis para el español», Revista CLAC (Círculo de Lingüística Aplicada a la Comunicación), 59, 148-162 [en línea]:
$<$ http://pendientedemigracion.ucm.es/info/circ ulo/no59/rgurillo.pdf>

RUIZ-GURILLO, Leonor (2016): «Variability, negotiability and adaptability in humorous monologues», Metapragmatics of humor: current research trends, Amsterdam, John Benjamins Publishing, 79-101, https://doi.org/10.1075/ivitra.14.05r ui

RUIZ GURILLO, Leonor (2017): «ldentifying feminine identity through Eva Hache's humorous monologues». Conferencia presentada en IPrA Conference, Belfast, 2017.

RuIz GuRILLO, Leonor, y Esther LINARES BERNABÉU (2018): «Shaping gender in Spanish stand-up comedy», conferencia presentada en ISHS conference, Tallin (Estonia).

RUTTER, Jason (2001): «Rhetoric in stand-up comedy: Exploring performer-audience interaction», Stylistyka, 10, 307-325.

ZADEH, Lofti (1965): «Fuzzy sets» Information and control, 8, 338-353.

ZIMMERMANN, Klaus (2003): «Anticortesía verbal y constitución de la identidad juvenil», en Bravo, Diana, ed., Actas del Primer Coloquio del Programa EDICE, La perspectiva no etnocentrista de la cortesía, identidad sociocultural de las comunidades hispanohablantes, Estocolmo, Universidad de Estocolmo, Departamento de español, portugués y estudios latinoamericanos, 47-59. 